adrenal gland but to produco a more dynamic approach to adrenal disease based on the knowledge of normal structure and function. In my opinion he has certainly succeeded.

The book commonces with a very large section on the adrenal cortex, describing features of the foetal, nconatal, infant and adult stages of this gland. The biosynthesis and metabolism of steroids are discussed, and the physiology and pathology of the cortex both in health and disease are more than adequately covered. Technical and chemical methods used for the investigations of disorders of the gland are slso given. I am pleased to see that the author aptly points out that investigations of the biosynthetic ability of adrenal tissue by radiochemical incubation studies in vitro need not reflect what is happening in vivo. More in vivo studies should certainly be made where possible.

The next section of the book deals with the adrenal medulla by A. M. Neville and again the same pattern of describing the foetal and adult gland followed by the biochemistry, physiology and pathology is followed as for the cortex section. An excellent chaptor on the detection and measurement of catecholamines in tissues and body fluids ends this section.

A section on the chemoreceptor system follows. At first this might seem to be out of context in a book about the adrenal gland, but the demonstration of catecholamines in the normal carotid body and in some tumours arising from it together with the possible neuroectodermal contribution to its development serves, I suppose, to emphasize the close relationship between the chemoreceptor and chromaffin systems.

A remarkable section devoted to an atlas of human adrenal cortex ultrastructure by A. Mackay now follows. This atlas, which is divided into three parts, gives well detailed electron micrographs of the three zones of the normal human cortex, then micrographs of the pathological human adrenal, and finally details of the processing techniques are given. Altogether more than a hundred micrographs are presented, which makes the book rather unique. Much of our present knowledge of steroid biosynthesis has been acquired from tissue slices or homogenates of normal and abnormal adrenals, and the electron microscope shows that all such preparations have a much greater structural heterogeneity than had been suspected by light microscopy. While the cells involved in normal and abnormal steroid biosynthesis have, however, been better characterized, many problems on the correlation of strueture and function of the adrenal cell still exist. It is nice to see that the major drawbacks of interpreting the electron micrographical data are pointed out.

The book finishes with a short section on the metabolic and hormonal response of the body to injury.

This book, though intended primarily for pathologists, will interest biochemists and endocrinologists. It seems rather expensive, but with its 550 pages with its excellent atlas of human cortex ultrastructure $I$ believe it to be well worth the money.

D. EXLEY

\section{DECOMPRESSION SICKNESS}

\section{Subatmospheric Decompression Sickness in Man}

By D. I. Fryer. (AGARDograph No. 125.) Pp. 343. (Technivision: Slough, 1969.) $145 s$.

IT is surprising to hear that in spito of all the advances in aircraft technology resulting in the provision of reliable pressure cabins and operational pressure suits, the hazards to man of subatmospheric decompression sickness have not decreased.

This book admirably illustrates the value of taking a critical overall look at a subject. It gets the problems into perspective and in this case reveals how fragmentary our knowledge is.

In an effort to determine precisely where we are in our undcrstanding of subatmospheric decompression sickness the author, who is a serving medical officer in the Royal Air Force, has assessed with remarkable perception a multitude of papors which have been written on this subject. In his evaluations he has made extensive use of his own practical experience and experimental observations. It is clear that we undoubtedly possess a lot of knowledge about decompression sickness but very little understanding, and the author rightly comes to the disappointing conclusion that "there can be few subjects as frustrating as that of subatmospheric decompression sickness, for its manifestations are so protean, its mechanisms so obscure and its prevention so difficult in practice".

For those who may wish to take up the challenge and to give serious consideration to this intriguing problem there is a wealth of informed opinion in this book and an excellent bibliography of more than 450 references.

\section{N. WALDER}

\section{JOINT ACTION}

\section{Lubrication and Wear in Joints}

Edited by Verna Wright. (Proceedings of a Symposium organized by the Biological Engineering Society and held at the General Infirmary, Leeds, on April 17, 1969.) Pp. 152. (Sector: London, March 1970. Distributed in the USA and Canada and South America by J. B. Lippincott.) 53s.

THIs collection of papers delivered at a symposium held about a year ago represents the thinking at that time of research groups, chiefly in the United Kingdom, on the subject of lubrication of diarthrodial joints. The newest theory, that of "trapped pool" lubrication, is given the greatest coverage. Observations on the structure of articular cartilage as interpreted by electron scanning are reported in some detail and excellent illustrations are provided in support. Those who support other theories of lubrication also had their say, both positively and, through the discussion, in challenging the validity of the data on which the trapped pool theory is based. A contribution on artificial lubricants, the last chapter, is spoiled by the omission of the table of criteria which a lubricant should fulfil before it could be used in an animal joint. Nor is there any evidence to support the premise that artificial lubricants could halt the disintegration of articular cartilage once fibrillation is established in weight-bearing areas.

The chapter on joint disease and the causation of osteoarthritis is disappointing in that it presents a standard list of causes which has been in use for many years and does not indicate that any new thinking about the subject is being indulged in by any of the groups represented at the meeting. There were no contributions dealing with the subject of wear of articular cartilage, but the impression is left that "wearing out" of cartilage as a purely mechanical process can be an important cause of osteoarthritis. It is evident from those papers that bioengineering research groups should have one member who is a biologist. Although the discussions applied some correctives there is no substitute for a built-in stabilizer.

Chapters are also devoted to joint prostheses and joint simulators-the problem of wear is a very real one when dealing with joint prostheses.

Elegant analyses of the forces acting on the hip and knee are presented by Paul. This work is the best dynamies study of joint forces so far available and must be required reading for everybody interested in joint function. We must also learn the lesson that the magnitude of force changes rapidly when the normal person is ambulant and is not accurately simulated by many of the compression studies carried out on cartilage to date.

As a deliberate policy an index has been dispensed with and bold marginal sub-headings have been used to indicate 